\title{
Juventud frente a la pandemia covid-19: el otro grupo en condición de vulnerabilidad ${ }^{1}$
}

\author{
Youth against the covid-19 pandemic: the other \\ group in a condition of vulnerability
}

\section{Alejandro Domínguez $* 1$}

Palabras clave: Jóvenes; Impacto; Pandemia; Covid-19; Salud mental.
Resumen: En la opinión pública, poco es tratado el impacto de la pandemia a causa del virus SARS-CoV-2 (COVID-19) sobre la juventud, aparentemente por el riesgo menor en la salud que este grupo de población tiene frente a otros grupos cuyo contexto y forma de vida aumentan la gravedad de la infección. Sin embargo, las consecuencias a mediano y largo plazo de los impactos en el desarrollo laboral, educativo, mental y emocional de las y los jóvenes, tiene un impacto que no se debe subestimar, sobre todo tomando en cuenta tanto el porcentaje y número de población juvenil en el mundo, y las condiciones preexistentes de las y los jóvenes que agravan estos impactos. Tanto los organismos internacionales, como los estudios realizados en la materia, coinciden en la necesaria atención a los efectos causados en la juventud por una crisis tan alargada, en la que no son inmunes de las consecuencias dañinas para su desarrollo. Por lo anterior, las decisiones públicas en las administraciones nacionales y locales, de la mano con la ciudadanía, deben replantear y enfocar la recuperación económica, laboral, social, emocional y educativa hacia la juventud, comenzando por no generalizar una supuesta inmunidad en este grupo poblacional, reconocer la vulnerabilidad en la que se encuentran expuesto, $y$, principalmente, dar espacio en las decisiones y aprovechar su emprendimiento y fortaleza en políticas y medidas sanitarias que eviten aumentar desigualdades entre generaciones, que anticipen y mitiguen impacto en la etapa posterior a la pandemia, y que den respuestas inclusivas a un problema público.

\footnotetext{
${ }^{1}$ Recebido em 03/06/2021. Aceito em 26/10/2021.

${ }^{*}$ Universidad de Salamanca. E-mail: alejandrodom94@gmail.com.
} 


\begin{abstract}
Keywords:
Abstract: In public opinion, the impact of the pandemic caused Youth;

Impact;

Pandemic;

Covid-19;

Mental Health. by the SARS-CoV-2 virus (COVID-19) on youth is little discussed, apparently due to the lower health risk that this population group has compared to other groups whose Context and lifestyle increase the severity of the infection. However, the medium and long-term consequences of the impacts on the labor, educational, mental and emotional development of young people, has an impact that should not be underestimated, especially taking into account both the percentage and number of the population. youth in the world, and the pre-existing conditions of young people that aggravate these impacts. Both international organizations and the studies carried out on the subject agree on the necessary attention to the effects caused on youth by such a prolonged crisis, in which they are not immune from the harmful consequences for their development. Therefore, public decisions in national and local administrations, hand in hand with citizens, must rethink and focus the economic, labor, social, emotional and educational recovery towards youth, beginning by not generalizing a supposed immunity in this group population, recognize the vulnerability in which they are exposed, and, mainly, give space in decisions and take advantage of their entrepreneurship and strength in health policies and measures that avoid increasing inequalities between generations, anticipating and mitigating impact in the post pandemic impact, and that provide inclusive responses to a public problem.
\end{abstract}

\title{
Introdução
}

El 11 de marzo de 2020, la Organización Mundial de la Salud (WHO, por sus siglas en inglés) declaró al SARS-CoV-2 (COVID-19, en lo sucesivo) como una pandemia (ORGANIZACIÓN MUNDIAL DE LA SALUD, 2020). En la evidencia actual, desde el inicio de la pandemia, uno de los mensajes definitorios ha sido que las y los jóvenes tienen menos riesgo de desarrollar síntomas graves de salud física relacionados con el Covid-19, a comparación de las personas adultas mayores. Sin embargo, personas de diferentes edades están experimentando sus efectos de diferentes maneras.

Los impactos sanitarios y no sanitarios de las y los jóvenes están demostrando ser significativos. Estas repercusiones del COVID-19 se extienden más allá de la salud y el bienestar, y más allá de la temporalidad de la pandemia. Tal como lo desarrolla el informe de la Organización de las Naciones Unidas (UN, por sus siglas en inglés) "Responsabilidad compartida, solidaridad global: una respuesta a los impactos socioeconómicos del COVID-19"2, esta

\footnotetext{
2 El informe describe la velocidad y la escala del brote, la gravedad de los casos y la interrupción social y económica de COVID-19. Véase GRUPO DE LAS NACIONES UNIDAS PARA EL DESARROLLO SOSTENIBLE (2020). Responsabilidad compartida, solidaridad global: Respuesta a los impactos socioeconómicos del COVID-19. Disponible en <https://unsdg.un.org/es/resources/responsabilidad-compartida-solidaridad-globalrespuesta-los-impactos-socioeconomicos-de>. Accedido en 1 de febrero de 2021.
}

Caderno Eletrônico de Ciências Sociais, Vitória, v. 9, n. 1, pp. 03-19, 2021. 
crisis mundial agrava vulnerabilidades y desigualdades existentes. Todos estos impactos son amplificados aún más en contextos donde se ha socavado la capacidad institucional y el acceso limitado a los servicios públicos y a los propios derechos humanos.

La presencia de factores como el sexo, el género, la nacionalidad, la discapacidad intelectual o física, o las desventajas socioeconómicas, pueden incrementar la vulnerabilidad de las y los jóvenes (incluidas las y los jóvenes que son lesbianas, gays, bisexual, transgénero, trasvesti, transexual, intersexual, queer, asexual, u otro [LGBTTTIQA+]; vagabundos, refugiados, maltratados, en rehabilitación de trastornos por uso de sustancias psicotrópicas, entre otras categorías). Por ello, merecen una consideración especial durante las atenciones derivadas del COVID-19.

A pesar de la heterogeneidad de este grupo, la pandemia COVID-19 expone tres agravios principales que comparten la mayoría de la juventud en todo el mundo: a) la pandemia afecta gravemente la salud física y mental; b) la "generación perdida"3 en donde se proyectan a las y los jóvenes, sufrirá las consecuencias educativas y económicas de la pandemia durante un tiempo considerable; $y, c)$ las y los jóvenes están sistemáticamente infrarrepresentados en el debate de las soluciones frente a la pandemia.

En este orden de ideas, el presente texto busca describir los riesgos de las y los jóvenes vulnerables, y reflexionar sobre las implicaciones de estos a largo plazo, para reflexionar si en verdad no constituyen un grupo o sector vulnerable; además pretende desarrollar la relación estrecha entre los impactos de la pandemia específicamente en las y los jóvenes, particularmente sus efectos en su salud mental a raíz de la situación laboral y profesional en la que se encuentran. Finalmente, se abordan reflexiones respecto a los desafíos a enfrentar en los próximos años desde la esfera social y gubernamental.

\section{Desarrollo}

Para las y los jóvenes, especialmente quienes se encuentran en situación de vulnerabilidad, la crisis por el COVID-19 presenta riesgos considerables en los campos de educación, el empleo, la salud mental y sus ingresos; riesgos de los que derivan consecuencias que posiblemente remplacen su bienestar largo plazo.

\footnotetext{
${ }^{3}$ El Grupo Asesor Científico para Emergencias (SAGE) de Reino Unido, han señalado que las y los niños y jóvenes corren el riesgo de convertirse en una "generación perdida" debido a las políticas antipandémicas. Véase HILL, A. (20 de octubre de 2020). 'Scarred for life': Sage experts warn of impact of Covid policies on the young [en línea]. The Guardian. Disponible en <https://amp.theguardian.com/uk-news/2020/oct/20/sage-experts-warn-ofimpact-of-covid-policies-on-young-generation-z-harm-pandemic-coronavirus $>$. Accedido en 1 de febrero de 2021.
}

Caderno Eletrônico de Ciências Sociais, Vitória, v. 9, n. 1, pp. 03-19, 2021. 
Somos la generación más grande de jóvenes: Mil 800 millones de personas entre 10 y 24 años en el mundo, de los cuales 165 millones se encuentran en América Latina y el Caribe, y 39.3 millones de personas entre 12 y 29 años en México (UN, 2020).

La juventud, como grupo de población específico, constituye una amplia gama de contextos, opiniones, privilegios y dificultades. Los impactos de COVID-19 son y seguirán siendo sentidos con más dureza por este grupo poblacional, que ya de por sí viven en situaciones difíciles o circunstancias desfavorecidas. Estos incluyen, pero no se limitan, a (COMPACT FOR YOUNG PEOPLE IN HUMANITARIAN ACTION, 2020):

- Personas jóvenes en situación de refugiadas, solicitantes de asilo o desplazados, o en alguna otra emergencia humanitaria preexistente.

- Jóvenes que viven en zonas urbanas pobres de alta densidad y asentamientos informales sin acceso o con poro acceso a servicios públicos elementales como agua potable y alcantarillado.

- Jóvenes con discapacidad, o con capacidad limitada para distanciarse físicamente, o con restricciones a su movimiento.

- Jóvenes con menos oportunidades de empleo.

- Personas jóvenes abandonadas o no acompañadas por padres o madres trabajadoras migrantes, que enfrentan mayor riesgo de explotación, violencia o problemas de salud mental.

- Mujeres jóvenes que ya sufren desigualdades y privaciones por razones de género, y corren un mayor riesgo de sufrir violencia de género.

- Jóvenes lesbianas, gays, bisexual, transgénero, trasvesti, transexual, intersexual, queer, asexual, u otro (LGBTTIIQA+), que tienden a enfrentar una discriminación generalizada, especialmente en entornos de atención médica.

- Jóvenes que enfrentan enfermedades crónicas.

- Jóvenes que viven con virus o enfermedades que debilitan su sistema inmunológico o que aumentan el riesgo de transmisión.

Pese a los números y realidades, a más de un año de declararse la pandemia a nivel mundial, la experiencia y evidencia médica ha demostrado que el COVID-19, además de afectar a personas adultas mayores, afecta también la salud física y mental de las y los jóvenes de muchas maneras: riesgo de ser afectados por el daño colateral de la pandemia; niveles de estrés más altos; trastornos psicosociales; entre otros (HILL, 2020; UNIVERSIDAD DE INNSBRUCK, 2020; GUESSOUM et al., 2020; LIANG et al., 2020).

De acuerdo con la Organización para la Cooperación y el Desarrollo Económicos (OECD, por sus siglas en inglés), una encuesta global de jóvenes de 15 a 24 años demuestra que las mayores preocupaciones que la pandemia les

Caderno Eletrônico de Ciências Sociais, Vitória, v. 9, n. 1, pp. 03-19, 2021. 
está causando son su salud mental, lo relacionado con su empleo, y la educación (OECD, 2020a).

Lo anterior cobra relevancia al partir de la asociación entre desempleo y la angustia psicológica y bienestar mental entre la juventud, que se encuentran fuertemente relacionadas. Se sabe que el desempleo, especialmente en escenarios de larga duración, tiene graves consecuencias en la salud física y mental de las personas desempleadas; estas consecuencias se refuerzan en medio de cualquier crisis, donde el desempleo es elevado y continuo, al tiempo que encienden alarmas de preocupación por la salud, informalidad e inactividad que traen consigo frustración y desaliento entre personas jóvenes, quienes se ven afectados también en sus familias y comunidades.

El acceso limitado de las y los jóvenes al mercado laboral, a raíz de la pandemia por el COVID-19, ha tenido consecuencias graves a corto y largo plazo. Principalmente, la crisis sanitaria ha impactado más allá de los indicadores laborales, pues ha afectado en buena medida el bienestar emocional de la juventud: las y los jóvenes que dejaron de trabajar o estudiar durante la pandemia tienen el doble de probabilidad de padecer ansiedad o depresión respecto a quienes siguieron en sus labores (ILO, 2020). Además, las estrictas restricciones sanitarias pueden aumentar conductas de riesgo, como el consumo de drogas y alcohol (WHO, 2002).

En ese sentido, el escenario laboral para las y los jóvenes no les es favorecedor. A finales del año 2019, la Organización Internacional del Trabajo (ILO, por sus siglas en inglés), indicó que en América Latina y el Caribe, habían 107.9 millones de jóvenes entre 15 y 24 años, de los cuales, 52.7 millones (48.9 por ciento) formaban parte de la fuerza de trabajo o tasa de participación laboral, mientras que el resto (55.2 millones de jóvenes) continuaban en actividades exclusivas de educación o estaban en situación de inactividad; cifra que disminuyó hasta un 17.9 por ciento a finales de ese año, señalando que 9.4 millones de jóvenes se encontraban en situación de desempleo en 2019. Pese a este avance, seis de cada diez jóvenes lograban insertarse en los mercados laborales en condiciones de informalidad, lo que propagaba condiciones de vulnerabilidad ante la pérdida de empleos e ingresos que inició meses después, en marzo 2020, desatada por la pandemia del COVID-19 (ILO, 2021). Por su parte, el Banco Mundial ha advertido que la pandemia puede costarle a la juventud diez billones de ingresos perdidos durante su vida, sin acciones decididas y coordinadas por parte de las administraciones públicas (BANCO MUNDIAL, 2020).

La dificultad de las y los jóvenes para una inserción laboral ha sido un problema constante en América Latina y el Caribe. La tasa de desempleo juvenil es tres veces la de los adultos; la tasa de informalidad es de 1.5 veces más

Caderno Eletrônico de Ciências Sociais, Vitória, v. 9, n. 1, pp. 03-19, 2021. 
alta; y la inactividad es 21 por ciento mayor en la juventud que no estudia, no se capacita o no trabaja (GONZÁLEZ VELOSA et. al., 2012; NOVELLA, 2018). EI COVID-19 ha dejado en esta región 17 millones de empleos perdidos, y la juventud ha sido la más afectada: En Perú, 70 por ciento de las y los jóvenes perdieron su empleo, mientras que en Colombia la tasa de desempleo juvenil pasó de 16 a casi 30 por ciento; y en República Dominicana, 37 por ciento de las personas menores de 24 años que tenían un empleo formal $(92$ mil personas) lo perdieron. Para el caso de México, más del 12 por ciento de las y los jóvenes empleados en el sector formal, perdieron su trabajo, porcentaje que representa casi 390 mil personas (OBSERVATORIO LABORAL COVID-19, 2021).

La dificultad de una adecuada inserción laboral de las y los jóvenes se pueden clasificar en cinco principales causas (MONTENEGRO, C. E. y PAGÉS, C., 2005; BERTRAND, M. et. al., 2004; ALAIMO, V., et. al., 2016; NOVELLA, R., et. al., 2018; CUNNINGHAM, W., 2009):

1) Aspectos regulatorios, que desfavorecen desproporcionadamente a las $y$ los jóvenes (por ejemplo, incrementos en el salario mínimo efectivo por encima del nivel de productividad);

2) Problemas de acceso y disponibilidad de información, lo que orillan a tomar la edad como indicador de productividad;

3) Incompatibilidad entre habilidades técnicas y socioemocionales de la juventud y las demandadas por los empleadores;

4) Desarticulación entre aspiraciones y realidad del mercado laboral (por ejemplo, en México, las y los jóvenes sobreestiman el salario esperado al egresar de la universidad); $y$,

5) Rotación elevada en el proceso de autoconocimiento profesional (las y los jóvenes de América Latina y el Caribe tienen en promedio 3.5 empleos en cuatro años de vida laboral).

La juventud que busca empleo también tiene menos posibilidades de regresar a sus lugares de trabajo, ya que muchos habían trabajado en áreas económicamente más afectadas por las restricciones (como bares, restaurantes, servicios de entretenimiento, ventas, educación y ocupaciones no profesionales). Las brechas de acceso al empleo se pronuncian de forma agravada por la pandemia COVID-19.

Estos problemas generan un efecto cicatriz (consecuencias graves a largo plazo más allá de la crisis actual). Existen estudios que demuestran que las condiciones al inicio de la vida profesional podrían tener efectos que permanezcan durante toda la trayectoria laboral, en términos de acceso, calidad e ingresos; asimismo, durante el desempleo, los ingresos para trabajadores jóvenes "poco calificados" puede den reducirse en más de 20 por ciento. Estos efectos pueden prevalecer incluso hasta por 15 años para aquellas personas que

Caderno Eletrônico de Ciências Sociais, Vitória, v. 9, n. 1, pp. 03-19, 2021. 
se gradúan e inician su vida laboral durante una recesión económica (KRAHN H. y CHOW, A., 2016; BELL, D. y BLANCHFL, D., 2010; CRUCES, G. et. al., 2012; GREGG, P., 2005; BURGESS, S., et. al. 2003; OYER, P., 2006).

En cuanto al impacto económico, numerosos análisis, anticipan que las y los jóvenes serán los más afectados por la recesión económica mundial que acompaña a la pandemia COVID-19. Incluso, de manera similar a la crisis financiera de 2009, se espera que aumenten el desempleo juvenil, la reducción de las oportunidades de vida, la pobreza y la deuda entre la juventud. En 2011, tras la crisis financiera referida, la ILO refirió que la las y los jóvenes fueron los primeros en perder su trabajo en tiempos de crisis económica y los últimos en obtener un empleo cuando la economía mejoraba (O'HIGGINS, 2011). En marzo de 2020, la UN mencionó que las y los jóvenes que ya estaban en un riesgo económico y social antes de la pandemia, ahora también están afectados de manera desproporcionada por el desempleo (UN, 2020); en el mismo sentido, la ILO ha manifestado que el déficit laboral formal se hará más evidente para ciertos grupos de trabajadores, como las y los jóvenes (ILO, 2020); por su parte la Organización para la Cooperación y el Desarrollo Económicos (OECD) ha señalado que las y los jóvenes tienen más probabilidades de caer en la pobreza (OECD, 2020b). Así, los organismos internacionales han trazado la línea clara de la situación preocupante de las y los jóvenes durante y después de la crisis financiera.

En un segundo ámbito, los efectos negativos a largo plazo se extienden a la salud emocional y física de las y los jóvenes hasta 14 años más tarde (HAMMARSTRÖM, A., y JANLERT. U., 2002). Estudios demuestran que los factores estresantes crónicos como el desempleo de larga duración tiene las consecuencias más negativas para la angustia psicológica de las personas, asociado con una mayor presión financiera y una mayor disminución de la autoestima. Además, podemos asumir que otros factores de estrés contextuales relacionados con el brote de COVID-19, como el miedo a infectarse, preocupaciones sobre familiares mayores, y las restricciones impuestas a nuestra vida social, también fortalecen el vínculo desempleo-angustia psicológica (ACHDUT, N., y REFAELI, T. 2020).

Con respecto a la edad, las personas más jóvenes tienen 6 por ciento más probabilidad de padecer trastornos mentales, siendo una posición más vulnerable respecto al de las personas adultas e, incluso, adultas mayores, en términos de salud mental en situación de aislamiento social (AHMED MZ, et. al., 2020).

He de resaltar también la desventaja de la población joven femenina, en la crisis de la salud mental ocasionada por la pandemia COVID-19. La ausencia de relaciones estables y desarrollo tardío del núcleo familiar, y las demandas

Caderno Eletrônico de Ciências Sociais, Vitória, v. 9, n. 1, pp. 03-19, 2021. 
socialmente impuestas al rol de la mujer, influyen en la prevalencia de psicopatologías entre las mujeres jóvenes. Incluso, el problema se agrava cuando los servicios de atención hospitalaria para las mujeres se ven interrumpidos y limitados para cuestiones tan esenciales como lo es el parto para mujeres jóvenes embarazadas, teniendo que recurrir a alternativas poco seguras e higiénicas, en medio de un contexto sanitario poco favorecedor4. Ante esta situación, se debe prestar especial atención a esta población, toda vez que existe una mayor recurrencia a desarrollar trastornos mentales, además del suicidio.

En este orden de ideas, las afectaciones al empleo y la salud mental, derivadas de la pandemia, amenazan con seguir impactando de manera profunda y a largo plazo la trayectoria laboral de las y los jóvenes, por lo que es necesario desarrollar políticas focalizadas a la juventud a fin de aminorar los efectos lo mayor posible. Por ello, las acciones de gobiernos de reactivación económica tendrán que fomentar y vigilar, tanto que las empresas garanticen condiciones laborales decentes para las y los jóvenes (así como implementar acciones afirmativas que beneficien específicamente a las mujeres por lo anteriormente señalado), como la atención médica y las medidas especiales de promoción de la salud entre las y los jóvenes desempleados, rompiendo el círculo vicioso del desempleo y la mala salud.

Los impactos en la educación también es un escenario sombrío. El desarrollo profesional de las y los jóvenes se ve afectada de forma desproporcionada en particular en su ámbito educativo, en el que sus programas de formación se encuentran interrumpidos y su emprendimiento se ve mermado. Sin duda, las y los jóvenes que abandonan la educación, temen perder oportunidades profesionales y educativas debido al impacto de la pandemia en la economía y su educación.

Los hallazgos de estudios realizados en el Reino Unido (Etheridge B., \& SPANTING L., 2020) y los Estados Unidos de América (MCGINTY EE. et al., 2020) muestran que las y los jóvenes experimentan un mayor nivel de angustia en comparación con otros grupos de edad. Además, la interrupción del proceso de aprendizaje en escuelas, derivada de la crisis sanitaria, ha deteriorado su bienestar; de acuerdo con la ILO, "los jóvenes cuya educación o trabajo se había interrumpido o había cesado totalmente tenían casi dos veces más probabilidades de sufrir probablemente ansiedad o depresión que los que continuaron trabajando o aquellos cuya educación siguió su curso" (ILO, 2020). Lo anterior pone en evidencia que existen vínculos entre el bienestar mental, la

\footnotetext{
${ }^{4}$ Ver en ese sentido LOREA, R. (2020, 1 de noviembre). La pandemia no tiene rostro de mujer. Disponible en <https://www.nexos.com.mx/?p=50896>. Accedido en 21 de marzo de 2021.
}

Caderno Eletrônico de Ciências Sociais, Vitória, v. 9, n. 1, pp. 03-19, 2021. 
continuación con los estudios educativos, y la integración de las y los jóvenes en el mercado de trabajo. Por lo anterior, será necesario restablecer de forma paulatina, mientras lo permitan las condiciones sanitarias, el cuidado de la salud de las y los jóvenes de manera presencial; ya que, volver a abrir y poner a disposición las prestaciones y servicios suspendidos, permitirá actuar de forma más organizada. Mientras tanto, es necesario mantener activos los canales de comunicación para convocar a las y los jóvenes a que retomen la atención en los servicios de salud.

De acuerdo con la ILO, 166 países han implementado cierres de escuelas y universidades en todo el país. Más de 1.52 millones de niñas, niños, adolescentes y jóvenes están actualmente fuera de la escuela o universidad, lo que representa el 87 por ciento de la población mundial de estudiantes (ONU, 2020 b, p. 3). Por otro lado, desde el comienzo de la pandemia, más del 70 por ciento de las y los jóvenes que estudian y trabajan, se han visto afectados gravemente por el cierre de escuelas y universidades, y 65 por ciento considera que su educación se ha visto afectada gravemente como consecuencia del inicio del periodo de educación a distancia o remota, durante el confinamiento; esto trae como consecuencia que el 9 por ciento de jóvenes considere abandonar sus estudios definitivamente (ILO, 2020b).

La situación se torna crítica para las y los jóvenes que viven en países de ingresos más bajos, en donde existen mayores deficiencias en materia de acceso a tecnologías e internet. Esto es, existe una brecha digital entre los países, pues mientras el 65 por ciento de las y los jóvenes de países de altos ingresos pueden asistir a clases impartidas por videoconferencia, sólo 18 por ciento de jóvenes en países de bajos ingresos pueden continuar con sus estudios vía remota. Para el caso mexicano, la última Encuesta Nacional sobre Disponibilidad y Uso de Tecnologías de la Información en los Hogares (ENDUTIH, por sus siglas) del año 2019, del Instituto Nacional de Estadística y Geografía (INEGI, 2019), muestra que 33 millones de hogares cuentan con televisión; y, por lo tanto, más de 1.6 millones de hogares no cuentan con este medio por el que se puede acceder a contenidos educativos del ciclo escolar. En cuanto al acceso a los materiales de enseñanza y propiamente las clases a través de internet, la EDUTIH señala que de las 80.6 millones de personas usuarias de internet, 8.5 millones de personas acceden a esta tecnología fuera del hogar, y de las 49.4 millones de personas usuarias de computadora, 14.5 millones de personas tienen acceso a éstas fuera de casa. Así, a pesar de que las y los jóvenes generalmente están muy conectados digitalmente, un gran número no tiene un acceso regular y asequible a internet y pueden quedarse atrás a medida que el aprendizaje y las plataformas educativas remotas cambian; además, hay que recordar, primeramente, que para las y los jóvenes, conectarse entre ellas y ellos es clave para un sentido de pertenencia $y$

Caderno Eletrônico de Ciências Sociais, Vitória, v. 9, n. 1, pp. 03-19, 2021. 
bienestar general; $y$, en segundo lugar, que el confinamiento y el aislamiento a largo plazo de los espacios educativos erosionan las redes de apoyo social que hemos construido.

Finalmente, más allá del problema de la vulnerabilidad preexistente de jóvenes, de los efectos inmediatos de la pandemia en la educación, salud y las oportunidades de vida de las y los jóvenes, existe también un tercer problema al que se enfrentan y que estimo necesario ni omitir referir, a saber, su falta de participación y nula consideración en los debates públicos y la subrepresentación en las tomas de decisiones en donde se acuerdan las políticas relacionadas con la pandemia. De acuerdo con la ILO, un tercio de las y los jóvenes percibió un impacto en su derecho a participar en los asuntos públicos (ILO, 2020b); en el mismo sentido, un estudio revela una pérdida de confianza en las instituciones públicas entre las y los jóvenes, particularmente a causa de quedar excluidos de los procesos de toma de decisiones en la pandemia (ANDRESEN, S. et al., 2020). A lo anterior le debemos sumar el contexto preexistente, donde, por ejemplo, en México, un 83.7 por ciento de las y los jóvenes, no están de acuerdo en que los políticos tomen en cuenta las ideas e inquietudes de los jóvenes (OIJ, 2019).

Pese a un escenario desolador de infrarrepresentación, se presenta un ánimo por parte de las y los jóvenes para movilizarse y hacer que se escuche su voz en la lucha contra la crisis sanitaria, a tal grado de que uno de cada cuatro jóvenes ha realizado algún tipo de trabajo voluntario durante la pandemia (ILO, 2020b). Por ejemplo, más de la mitad de las organizaciones dirigidas por jóvenes, han realizado algún tipo de aporte para ayudar a los jóvenes a lidiar con el estrés mental (OECD, 2020). La participación de las y los jóvenes en la toma de decisiones armonizadas a sus necesidades y proyectos aumenta la eficacia de las políticas y los programas y les brinda oportunidad de contribuir a su aplicación.

\section{Conclusiones}

Al referir los efectos e impactos causados por la pandemia del virus COVID-19, se suele pensar en que la mayoría de las y los jóvenes pueden evadir un caso grave de afectación, cuando en realidad no tienen tanta suerte en otros ámbitos como lo es su empleo, educación, y, consecuentemente, su salud mental durante la pandemia.

Los impactos en la salud y economía por la pandemia COVID-19 han sido asimétricos en todos los grupos de edad. Pese a que, hasta ahora, hemos exiliado a la juventud como grupo vulnerable en los impactos sanitarios, los efectos de la pandemia en las y los jóvenes es sistemática, profunda y desproporcionada.

Caderno Eletrônico de Ciências Sociais, Vitória, v. 9, n. 1, pp. 03-19, 2021. 
Este contexto se vuelve aún más preocupante, ya que la juventud es sólo una de las muchas categorías de discriminación y marginación. La exclusión, por ejemplo, por discapacidad o sexualidad, requieren una atención especial, pues, como se ha desarrollado, la pandemia vino, además, a aumentar desigualdades existentes.

Una crisis sanitaria tan alargada como la ocasionada por la pandemia COVID-19, que retrasa la recuperación económica e impacta de manera directa a la salud mental, a causa de las estrictas medidas de confinamiento para enfrentarla, afecta de forma desproporcionada a las y los jóvenes, impactando en sus expectativas, aspiraciones y productividad. La nueva normalidad deberá considerar medidas que indiquen la ruta hacia soluciones para problemas de empleo y bienestar emocional juvenil. A pesar de que la juventud tenga menos probabilidades de una afectación grave al momento de enfermarse, no les hace inmunes a las consecuencias tan dañinas como lo es las afectaciones al bienestar mental. Las pérdidas asociadas con el desempleo pueden conducir al aislamiento social; de hecho, el desempleo juvenil, junto con la pérdida de los lazos y las interacciones sociales diarias y regulares (que proporcionan un sentido de pertenencia y apoyo), se ha encontrado relacionado con la soledad, y esto implica que los desempleados de larga duración soporten una carga de enfermedad mental notablemente mayor en comparación con las personas empleadas y los que están desempleados por poco tiempo, y la carga de enfermedad aumenta con la duración de los desempleados.

Por otra parte, la transición de escuela al trabajo es una etapa fundamental para el desarrollo de la persona, un momento crucial de la vida juvenil; y de ese tamaño es la necesidad de apoyar a las y los jóvenes para poder enfrentar con éxito los obstáculos y retos en esta etapa generados por la falta de experiencia profesional, menor número de empleos disponibles, interrupción de educación o formación, y urgencia de generar ingresos.

Asimismo, la pandemia hizo más visible la vulnerabilidad de la juventud en el ámbito laboral, con empresas a las que se les facilita recortar personal con menor experiencia o, incluso, derechos. El acceso no adecuado de las y los jóvenes al mercado laboral, a causa del COVID-19, genera consecuencias graves a largo plazo, que se extenderán incluso después de la recuperación económica. Una sociedad que no apuesta a renovarse, creando empleos dignos para sus jóvenes y apoyando a las pequeñas, medianas y grandes empresas para la inserción de jóvenes, es una sociedad condenada al envejecimiento con improductividad y bajo crecimiento.

Lo anterior, hace inminente que las y los hacedores de políticas públicas, en conjunto con las universidades, implementen medidas para mitigar dichas consecuencias; para ello, es fundamental fomentar trayectorias laborales

Caderno Eletrônico de Ciências Sociais, Vitória, v. 9, n. 1, pp. 03-19, 2021. 
exitosas por medio de acciones como incentivos a la contratación de jóvenes, formación y capacitación de habilidades según las nuevas realidades del mercado laboral, y, desde luego, apoyo a la búsqueda de empleo. De no ser así, toda una generación (que, dicho sea de paso, es la columna vertebral del crecimiento social y económico de las regiones) se vea afectada por un tiempo prolongado, simplemente por haber tenido el error de egresar de la universidad en un momento no idóneo como lo es la pandemia por COVID-19. Procurar un trabajo estable y digno para las y los jóvenes es vital, pues de ello depende que se garantice la sobrevivencia de la propia sociedad en su conjunto.

Por lo anterior, existe una necesidad real de que las y los jóvenes, como afectados a corto y largo plazo, no se generalice y no sean reconocidos ni como víctimas ni, mucho menos, como aceleradores de la pandemia o irresponsables, rebeldes e infractores de las medidas de control y contención; sino como agentes y fuentes de inspiración en políticas y medidas sanitarias.

Sus contribuciones y prácticas para mitigar el impacto de la pandemia han buscado un espacio entre los debates sobre las respuestas globales en el contexto sanitario de COVID-19. Al no encontrar lugar en estos, las respuestas de autoridades y gobiernos deben tornarse inclusivas, ya que las recuperaciones de las crisis requieren un enfoque integrado de la gobernanza pública que anticipe el impacto de las medidas de respuestas y recuperación en diferentes grupos de edad. Así, es necesario que quienes tomen decisiones reconozcan a las generaciones, y las atiendan, de tal forma que nadie quede atrás.

Por lo anterior, las administraciones públicas deben desarrollar y fortalecer iniciativas de salud con el objeto de aliviar las consecuencias para la salud mental ocasionadas por el desempleo en tiempos de pandemia. Estas medidas deben considerarse como la ruta para promover la salud pública, cuyo enfoque principal sea aliviar la soledad, en particular de las y los jóvenes que no han encontrado sus trabajos o, con mayor razón de quienes los han perdido en medio de la pandemia.

En conclusión, para evitar aumentar las desigualdades entre generaciones e involucrar a las y los jóvenes en la construcción de las propuestas a problemas sociales de gran impacto, los gobiernos deben anticipar el impacto de las medidas que combaten o previenen el COVID-19, primeramente, reconociendo a la juventud como grupo vulnerable por los impactos en su salud, educación y desarrollo profesional, y, consecuentemente, aplicando mecanismos de gobernanza efectivos, en los que es fundamental que se escuche la voz de las y los jóvenes para dar respuestas más inclusivas a la crisis sanitaria actual.

Caderno Eletrônico de Ciências Sociais, Vitória, v. 9, n. 1, pp. 03-19, 2021. 


\section{Bibliografía}

ACHDUT, N., y REFAELI, T. 2020. Unemployment and Psychological Distress among Young People during the COVID-19 Pandemic: Psychological Resources and Risk Factors, en International Journal of Environmental Research and Public Health, vol. 17, n. 19, 30 de septiembre de 2020, pp. $7163 . \quad$ Disponible en <https://www.mdpi.com/16604601/17/19/7163/htm>. Accedido en 21 de marzo d 2021.

AHMED MZ, et. al. 2020. Epidemic of COVID-19 in China and Associated Psychological Problems. Asian Journal of Psychiatry, vol. 51. Disponible en https://www.sciencedirect.com/science/article/abs/pii/S1876201820302033 $>$. Accedido en 25 de febrero de 2021.

ALAIMO, V., et. al., 2016. Empleos para Crecer. Banco Interamericano de Desarrollo.

ANDRESEN, S. et al. 2020. Erfahrungen und Perspektiven von jungen Menschen während der Corona-Maßnahmen. (Experiencias y perspectivas de los jóvenes durante las medidas del coronavirus). Disponible en https://hildok.bsz-bw.de/frontdoor/index/index/docId/1078 Accedido en 2 de febrero del 2021.

BANCO MUNDIAL. 2020. COVID-19 Could Lead to Permanent Loss in Learning and Trillions of Dollars in Lost Earnings. Disponible en <https://www.worldbank.org/en/news/press-release/2020/06/18/covid-19could-lead-to-permanent-loss-in-learning-and-trillions-of-dollars-in-lostearnings $>$. Accedido en 25 de febrero de 2021].

BELL, D. y BLANCHFL, D. 2010. Youth Unemployment: Déjà Vu?. IZA Discussion Papers 4705, Institute of Labor Economics (IZA). Disponible en <http://ftp.iza.org/dp4705.pdf>. Accedido en 25 de febrero de 2021.

BERTRAND, M., et. al. 2004. A Behavioral-Economics View of Poverty, en American Economic Review, vol. 94, No. 2, pp. 419-423.

BURGESS, S., et. al. 2003. The class of 1981: the effects of early career unemployment on subsequent unemployment experiences, en Labour Economics, vol. 10, n. 3, pp. 291-309. Disponible en <https://www.sciencedirect.com/science/article/abs/pii/S092753710200138 $0>$ Accedido en 25 de febrero de 2021.

COMPACT FOR YOUNG PEOPLE IN HUMANITARIAN ACTION. 2020. COVID-19: Working with and for young people. Disponible en $<$ https://reliefweb.int/sites/reliefweb.int/files/resources/76623.pdf >.

Accedido en 1 de febrero de 2021.

CRUCES, G. et. al. 2012. "Scarring effects of youth unemployment and informality Evidence from Brazil". Working Paper 8017, Centre for Distributive, Labor and Social Studies, IZA, Bonn, Alemania. Disponible en

Caderno Eletrônico de Ciências Sociais, Vitória, v. 9, n. 1, pp. 03-19, 2021. 
<http://conference.iza.org/conference_files/worldb2012/viollaz_m8017.pdf $>$. Accedido en 25 de febrero de 2021.

CUNNINGHAM, W. 2009. "Unpacking youth unemployment in Latin America," Policy Research Working Paper Series 5022, The World Bank. Disponible en <http://documents1.worldbank.org/curated/en/591521468147840898/pdf/ WPS5022.pdf > . Accedido en 25 de febrero de 2021.

ETHERIDGE B., \& SPANTING L. 2020, The Gender Gap in Mental Well-Being During the Covid-19 Outbreak: Evidence from the UK. ISER Working Paper Series 2020-08, Institute for Social and Economic Research. Disponible en <https://www.iser.essex.ac.uk/research/publications/workingpapers/iser/2020-08>. Accedido en 1 de febrero de 2021.

GONZÁLEZ-VELOSA, C., et. al. 2012. ¿Cómo mejorar las oportunidades de inserción laboral de los jóvenes en América Latina? Banco Interamericano de Desarrollo. Disponible en $<$ https://publications.iadb.org/publications/spanish/document/\%C2\%BFC\% C3\%B3mo-mejorar-las-oportunidades-de-inserci\%C3\%B3n-laboral-de-losj\%C3\%B3venes-en-Am\%C3\%A9rica-Latina.pdf>. Accedido en 25 de febrero de 2021.

GREGG, P. 2005. The wage scar from male youth unemployment, en Labour Economics, Volumen 12, Número 4, Agosto 2005, Pp. 487-509. Disponible en

<https://www.sciencedirect.com/science/article/abs/pii/S092753710500034 5? via\%3Dihub>. Accedido en 25 de febrero de 2021.

GRUPO DE LAS NACIONES UNIDAS PARA EL DESARROLLO SOSTENIBLE. 2020. Responsabilidad compartida, solidaridad global: Respuesta a los impactos socioeconómicos del COVID-19. Disponible en $<$ https://unsdg.un.org/es/resources/responsabilidad-compartidasolidaridad-global-respuesta-los-impactos-socioeconomicos-de $>$. Accedido en 1 de febrero de 2021.

GUESSOUM et al. 2020. Adolescent psychiatric disorders during the COVID-19 pandemic and lockdown, Disponible en <https://www.ncbi.nlm.nih.gov/pmc/articles/PMC7323662/>. Accedido en 1 de febrero de 2021.

HAMMARSTRÖM, A., Y JANLERT. U. 2002. Early unemployment can contribute to adult health problems: results from a longitudinal study of school leavers, en Journal Epidemiol Community Health, n. 56, pp. 624-630. Disponible en $<$ https://jech.bmj.com/content/jech/56/8/624.full.pdf>. Accedido en 25 de febrero de 2021.

HILL, A. (2020). 'Scarred for life': Sage experts warn of impact of Covid policies on the young [en línea]. The Guardian. Disponible en <https://amp.theguardian.com/uk-news/2020/oct/20/sage-experts-warn-

Caderno Eletrônico de Ciências Sociais, Vitória, v. 9, n. 1, pp. 03-19, 2021. 
of-impact-of-covid-policies-on-young-generation-z-harm-pandemiccoronavirus>. Accedido en 1 de febrero de 2021.

INSTITUTO NACIONAL DE ESTADÍSTICA Y GEOGRAFÍA (INEGI) (2019). Encuesta Nacional sobre Disponibilidad y Uso de Tecnologías de la Información en los Hogares (ENDUTIH 2019). Disponible en <https://www.inegi.org.mx/programas/dutih/2019/> Accedido en 1 de febrero de 2021.

KRAHN H. y CHOW, A., (2016). Youth Unemployment and Career Scarring: Social-Psychological Mediating Effects?, en Canadian Journal of Sociology, vol. $41, \quad n . \quad 2 . \quad$ pp. 117-138. Disponible en $<$ https://journals.library.ualberta.ca/cjs/index.php/CJS/article/view/23863/ 20545> Accedido en 25 de febrero de 2021.

LIANG, L. et al. 2020. The Effect of COVID-19 on Youth Mental Health. Psychiatr Q 91, pp. 841-852.

LOREA, R. 2020. La pandemia no tiene rostro de mujer. Disponible en $<$ https://www.nexos.com.mx/?p=50896> Accedido en 21 de marzo de 2021.

MCGINTY, E. et al. (2020, 3 de junio), Psychological Distress and Loneliness Reported by US Adults in 2018 and April 2020. JAMA. 2020;324(1). Pp. 9394. Disponible <https://jamanetwork.com/journals/jama/fullarticle/2766941> Accedido en 1 de febrero de 2021.

MONTENEGRO, C. E. y PAGÉS, C. 2005. "Who Benefits from Labor Market Regulations? Chile 1960-1998," Central Banking, Analysis, and Economic Policies Book Series, en: Restrepo, J \& Tokman R, A. \& Loayza, N. (Series Editor) \& Klaus Schmidt-Hebbel (Series Edi (ed.), Labor Markets and Institutions, ed. 1, vol. 8, cap. 4, pp. 77-114.

NOVELLA, R., et. al., 2018. Millennials en América Latina y el Caribe: ¿trabajar o estudiar? Banco Interamericano de Desarrollo. Disponible en $<$ https://publications.iadb.org/publications/spanish/document/Millennials_e n_Am\%C3\%A9rica_Latina_y_el_Caribe_trabajar_o_estudiar.pdf> Accedido en 25 de febrero de 2021.

O'HIGGINS, N. 2011. The impact of the economic and financial crisis on youth employment: Measures for labour market recovery in the European Union, Canada and the United States. ILO Working Papers 994621293402676, International Labour Organization. Disponible en <https://www.ilo.org/employment/Whatwedo/Publications/workingpapers/WCMS_154365/lang--en/index.htm> Accedido en 1 de febrero de 2021.

OBSERVATORIO DE LA JUVENTUD EN IBEROAMÉRICA (OJI). 2019. Encuesta de jóvenes en México 2019. México: Fundación SM. Disponible en <https://www.observatoriodelajuventud.org/encuesta-mexicana-de-la-

Caderno Eletrônico de Ciências Sociais, Vitória, v. 9, n. 1, pp. 03-19, 2021. 
juventud/\# : :text=La\%20Encuesta\%20de\%20j\%C3\%B3venes,juveniles $\% 2$ Oen\%20toda\%20la\%20regi\%C3\%B3n>. Accedido en 2 de febrero de 2021 . OBSERVATORIO LABORAL COVID-19. 2021. Informe Periódico Enero 2021: EI Impacto de la Covid-19 en el Mercado Laboral de América Latina. Banco Interamericano de Desarrollo. Disponible en <https://observatoriolaboral.iadb.org/es/assets/pub_obslab/Informe_OLC_2 02101.pdf> Accedido en 25 de febrero de 2021.

ORGANIZACIÓN MUNDIAL DE LA SALUD (WHO). 2020. Alocución de apertura del Director General de la WHO en la rueda de prensa sobre la COVID-19 celebrada el 11 de marzo de 2020. Disponible en <https://www.who.int/es/director-general/speeches/detail/who-directorgeneral-s-opening-remarks-at-the-media-briefing-on-covid-19---11-march2020>. Accedido en 1 de febrero de 2021.

ORGANIZACIÓN MUNDIAL DE LA SALUD (WHO). 2002. World Report on Violence and Health. Disponible en <https://www.who.int/violence_injury_prevention/violence/world_report/en /full_en.pdf?ua=1> Accedido en 25 de febrero de 2021.

ORGANIZACIÓN PARA LA COOPERACIÓN Y EL DESARROLLO ECONÓMICOS (OECD). 2020a. Youth And Covid-19: Response, Recovery And Resilience. Disponible en <https://read.oecd-ilibrary.org/view/?ref=134_134356ud5kox3g26\&title=Youth-and-COVID-19-Response-Recovery-and-

Resilience>. Accedido en 24 de febrero de 2021.

ORGANIZACIÓN PARA LA COOPERACIÓN Y EL DESARROLLO ECONÓMICOS (OECD). 2020b. COVID-19: Protecting people and societies. Disponible en https://www.oecd.org/coronavirus/en/. Accedido en 1 de febrero de 2021.

ORGANIZACIÓN DE LAS NACIONES UNIDAS (UN). 2020. Presentan Mirada Joven, una plataforma para que las y los jóvenes tomen decisiones basados en información confiable. Disponible en <https://www.onu.org.mx/presentan-mirada-joven-una-plataforma-paraque-las-y-los-jovenes-tomen-decisiones-basados-en-informacionconfiable/>. Accedido en 1 de febrero de 2021.

ORGANIZACIÓN DE LAS NACIONES UNIDAS (UN). 2020b. Special issue on COVID-19 and Youth. Disponible en $<$ https://www.un.org/development/desa/dspd/wpcontent/uploads/sites/22/2020/04/YOUTH-FLASH-Special-issue-on-COVID19-1.pdf>. Accedido en 1 de febrero de 2021. ORGANIZACIÓN INTERNACIONAL DEL TRABAJO (ILO). 2020. Los jóvenes y la pandemia de la COVID-19: efectos en los empleos, la educación, los derechos $y$ el bienestar mental. Disponible en $<$ https://www.ilo.org/global/topics/youth- 
employment/publications/WCMS_753054/lang--es/index.htm>. Accedido en 24 de febrero de 2021.

ORGANIZACIÓN INTERNACIONAL DEL TRABAJO (ILO). 2020b. Labour Overview in times of COVID-19: Impact on the labour market and income in Latin America and the Caribbean. Second Edition. Disponible en $<$ https://www.ilo.org/wcmsp5/groups/public/---americas/---rolima/documents/publication/wcms_756697.pdf>. Accedido en 1 de febrero de 2021.

ORGANIZACIÓN INTERNACIONAL DEL TRABAJO (ILO). 2021. World Employment and Social Outlook: Trends 2021. Ginebra. International Labour Organization 2021.

OYER, P. 2006. Initial Labor Market Conditions and Long-Term Outcomes for Economists, en Journal Of Economic Perspectives, Volumen 20, Número 3, 2006, pp. 143-160. Disponible en <https://www.aeaweb.org/articles?id=10.1257/jep.20.3.143>. Accedido en 25 de febrero de 2021.

UNIVERSIDAD DE INNSBRUCK (2020, 28 de septiembre). Projekte. Alemania: Forschungszentrum Gesundheit und Prävention über die Lebensspanne. Disponible en <https://www.uibk.ac.at/fz-gesundheitpraevention/projekte.html>. Accedido en 1 de febrero de 2021. 